\title{
Termorregulación de la lagartija Liolaemus chacoensis (Squamata: Liolaemidae) durante su ciclo reproductivo, en el Chaco occidental, Argentina
}

\author{
Graciela Astudillo 1,2,4*, Mariela Córdoba 1,2,4, Rodrigo Gómez Alés ${ }^{2,3,4}$, Juan Carlos Acosta 1,3,4 \\ \& Héctor José Villavicencio ${ }^{3,4}$ \\ 1. Centro de Investigaciones de la Geósfera y Biosfera (CIGEOBIO)-CONICET. Facultad de Ciencias Exactas, Físicas \\ y Naturales, Universidad Nacional de San Juan, Av. Ignacio de la Roza 590, San Juan, Argentina; \\ astudillo.vanesa@gmail.com, carranzamari@gmail.com, jcacostasanjuan@gmail.com \\ 2. Consejo Nacional de Investigaciones Científicas y Técnicas (CONICET), Argentina; rodri.gomezales@gmail.com \\ 3. Departamento de Biología, Facultad de Ciencias Exactas, Físicas y Naturales, Universidad Nacional de San Juan, San \\ Juan, Argentina; hjvillavicencio@gmail.com \\ 4. Gabinete de Diversidad y Biología de Vertebrados del Árido (DIBIOVA-UNSJ). Facultad de Ciencias Exactas, Físicas \\ y Naturales, Universidad Nacional de San Juan, San Juan, Argentina. \\ * Correspondencia
}

Recibido 08-I-2019. Corregido 14-VIII-2019. Aceptado 01-X-2019.

\begin{abstract}
Thermoregulation of Liolaemus chacoensis lizard (Squamata: Liolaemidae) during its reproductive cycle, in the Western Chaco, Argentina. Regulation of body temperature in lizards plays a key role in the survival of species. Their physiological condition, in reference to their dependence on the ambient temperature makes them an excellent study model. Also, changes in the reproductive cycle cause changes in thermophysiological parameters. This study is the first to address the relationship of thermoregulation with reproductive activity and sex, for a species of Liolaemus from the Chaco ecoregion. The aim of this work was to determine how the reproductive, recruitment and post-reproductive periods influences, as well as the effect of sex and snout-vent length on the thermoregulation of Liolaemus chacoensis. Individuals were captured by hand in the Southern sector of Valle Fertil, San Juan province, Argentina. A total of 44 individuals were captured during the months of November 2014, February 2015 and March 2015. Body $\left(\mathrm{T}_{\mathrm{b}}\right)$, substrate $\left(\mathrm{T}_{\mathrm{s}}\right)$ and air $\left(\mathrm{T}_{\mathrm{a}}\right)$ temperatures were recorded. Also, biophysical models were used to record operative temperatures. Later selected temperatures $\left(T_{\text {sel }}\right)$ were evaluated at the laboratory. Finally, thermoregulatory efficiency index was calculated. Results indicated that L. chacoensis $\mathrm{T}_{\mathrm{b}}$ was positively related to $\mathrm{T}_{\mathrm{a}}$, presenting a heliothermic behavior that was maintained during periods and sex. Also, $\mathrm{T}_{\mathrm{b}}$ was higher in reproductive and recruitment periods than in post-reproductive period. In particular, only sex had an effect on $\mathrm{T}_{\text {sel}}$, however for the interquartile range of $\mathrm{T}_{\text {sel }}$ both the period of reproductive cycle and sex had an effect. The thermoregulatory efficiency index indicated that $L$. chacoensis is an absolute thermoconformist. However, in the reproductive period both males and females change towards active thermoregulation. These results show that thermophysiological parameters vary in different periods of reproductive cycle and the active microsite selection would be a mechanism in maintenance of body temperature of this species.
\end{abstract}

Key words: temperature, effectiveness of thermoregulation, thermal quality, ectotherms, reproductive cycle.

Astudillo, G., Córdoba, M., Gómez Alés, R., Acosta, J. C., \& Villavicencio, H. J. (2019) Termorregulación de la lagartija Liolaemus chacoensis (Squamata: Liolaemidae) durante su ciclo reproductivo, en el Chaco occidental, Argentina. Revista de Biología Tropical, 67(6), 1505-1519. 
La regulación de la temperatura corporal en los lagartos cumple un rol clave para la supervivencia de las especies. Su condición fisiológica, en referencia a su dependencia de la temperatura ambiente, los convierte en un excelente modelo de estudio. Los lagartos combinan una serie de respuestas conductuales, bioquímicas y fisiológicas (Marquet, Ortíz, Bozinovic, \& Jaksic, 1989) que aseguran que su temperatura corporal esté dentro de los límites máximos y mínimos de su nicho térmico (Moyes \& Schulte, 2007). Asimismo, los lagartos obtienen el calor a través de la radiación directa del sol (heliotermia) o por contacto con el sustrato (tigmotermia) (Martori, Aun, \& Orlandini, 2002; Pianka \& Vitt, 2003). Además, algunos factores intrínsecos como el sexo, tamaño y masa corporal afectarían el intercambio calórico, y pueden ayudar a una mayor eficiencia en la termorregulación (Stevenson, 1985; Christian, 1998; Du, Yan, \& Ji, 2000). Las estrategias de termorregulación, dependen de la forma en la cual obtienen el calor, siendo termoconformista cuando su temperatura corporal es similar a las que le provee el ambiente sin modificarla. A su vez, otro tipo de estrategia es la termorregulación activa, donde el individuo mantiene su temperatura corporal a una temperatura diferente a las de su ambiente, a través de una variedad de mecanismos conductuales y fisiológicos (Huey \& Slatkin, 1976; Zug, Vitt, \& Caldwell, 2001). Para determinar las estrategias termorregulatorias, Hertz, Huey y Stevenson (1993) plantean la necesidad de conocer no sólo la temperatura corporal de campo sino también la temperatura (o el rango intercuartil de temperaturas) que seleccionan en ausencia de factores bióticos y abióticos; que es denominada temperatura seleccionada $\left(\mathrm{T}_{\text {sel }}\right)$ (Hertz et al., 1993). A su vez, estos autores destacan la importancia de conocer las temperaturas operativas; que representan la distribución nula de las temperaturas corporales potenciales que un animal no termorregulador podría experimentar en el ambiente de estudio (Hertz et al., 1993). Este método ha facilitado la caracterización cualitativa y cuantitativa de la termorregulación en diversas especies de lagartijas, en estudios de selección de hábitat (Bauwens, Hertz, \& Castilla, 1996; Christian \& Weavers, 1996; Grbac \& Bauwens, 2001; Sartorius, do Amaral, Durtsche, Deen, \& Lutterschmidt, 2002), partición de recursos (Hertz, 1992) y en los que han abordado el efecto de la estacionalidad en los mecanismos conductuales de termorregulación (Bauwens et al., 1996; Díaz \& Cabezas-Díaz, 2004; Stellatelli et al., 2018).

El género Liolaemus está representado por más de 257 especies que se distribuyen a lo largo de una amplia diversidad de ambientes en América del Sur (Abdala \& Quinteros, 2014). Este género de lagartos es ecológicamente versátil, las especies muestran diferencias intra e interespecífica en las temperaturas corporales de campo, en el comportamiento de termorregulación y en los patrones de actividad diaria asociada a las condiciones climáticas (Fuentes \& Jaksic, 1979; Jaksic \& Schwenk, 1983; Marquet et al., 1989; Carothers, Marquet, \& Jaksic, 1998; Labra, 1998; Martori et al., 2002; Medina et al., 2012; Moreno Azócar et al., 2013; Cruz et al., 2014). En los últimos años ha sido un grupo muy estudiado, en el cual se ha reportado información sobre su ecología, fisiología y otros aspectos de la biología (Abdala \& Quinteros, 2014). En base a estos estudios se ha sugerido que las temperaturas corporales de campo dependen en gran medida de las temperaturas ambientales (Labra, Vidal, Solis, \& Penna, 2008) y que Liolaemus tendría una fisiología térmica flexible, como es el caso de las poblaciones de L. bellii estudiadas por Labra (1998). Particularmente, este estudio es el primero en abordar en una especie de Liolaemus de la ecorregión del Chaco, la relación de la termorregulación con la actividad reproductiva y el sexo. Esta región es un área subtropical que presenta diferencias estacionales bien marcadas (Sarmiento, 1972; Díaz, 2007) y los valores promedios de temperaturas ambientales son variables (Karlin, 2012). Liolaemus chacoensis es la especie más representativa del bosque chaqueño, se distribuye desde latitudes tropicales $\left(21^{\circ} \mathrm{S}\right)$ (Gonzales, Muñoz, \& Cortez, 2006; Aguilar-Kirigin \& Ocampo, 
2011) hasta los $33^{\circ}$ de latitud sur (Fitch, 1970). $\mathrm{Su}$ distribución abarca las áreas chaqueñas de Paraguay, Argentina (Cei, 1993; Lavilla, Cruz, \& Scrocchi, 1995; Uetz, 2006) y Bolivia (Gonzales et al., 2006; Aguilar-Kirigin \& Ocampo, 2011). En Argentina, se distribuye en sectores del Chaco Árido de Llanura y Chaco Serrano. Es un lagarto pequeño, no supera los $55 \mathrm{~mm}$ de longitud hocico-cloaca y las hembras son de mayor tamaño (47.2 mm LHC) que los machos (42.3 mm LCH) (Cabrera, Scrocchi \& Cruz, 2013; Astudillo, 2018). En lagartos de ambientes templados, los ciclos reproductivos responden a un patrón estacional (CruzElizalde \& Ramírez-Bautista, 2016; Stellatelli et al., 2018). La duración de cada evento del ciclo reproductivo, tales como gametogénesis, ovulación, cópula, fertilización, oviposición y nacimientos, está regulado por la temperatura ambiental (Ortiz, 1981; Olivares, Tapia, Estica, Henriquez, \& Bustos Obregon, 1987, Pough et al., 1998). En particular, para una población de $L$. chacoensis de zonas templadas y de baja altitud, Cruz y Ramírez Pinilla (1996) mencionan que es una especie ovípara con un ciclo reproductivo afectado por la temperatura ambiental, describiendo así un patrón de actividad reproductiva estacional, de primavera-verano. En este contexto, se plantearon tres interrogantes: primero, ¿los diferentes períodos del ciclo reproductivo afectan la termorregulación de L. chacoensis?, segundo, ¿existen diferencias sexuales en la termorregulación de $L$. chacoensis? y tercero, ¿la longitud hocico-cloaca (LHC) influye en la termorregulación? En este trabajo, los objetivos propuestos fueron: (1) determinar la relación entre la temperatura del aire $\left(\mathrm{T}_{\mathrm{a}}\right)$ y la temperatura del sustrato $\left(\mathrm{T}_{\mathrm{s}}\right)$ con la temperatura corporal de campo $\left(\mathrm{T}_{\mathrm{c}}\right)$ de $L$. chacoensis, probando el efecto de la LHC, del período (reproductivo, reclutamiento y posreproductivo) y sexo; (2) contrastar la $\mathrm{T}_{\mathrm{c}}, \mathrm{T}_{\mathrm{a}} \mathrm{y}$ $\mathrm{T}_{\mathrm{s}}$ según el período y el sexo; (3) contrastar la $\mathrm{T}_{\text {sel }}$ según el período, sexo y LHC; (4) conocer la variabilidad de las temperaturas operativas en tres períodos del ciclo reproductivo y en cinco micrositios, en un sector del extremo sur del Chaco occidental y (5) cuantificar y comparar los índices descriptivos de termorregulación (precisión en la termorregulación, calidad térmica del hábitat y eficiencia en la termorregulación) de acuerdo al período y sexo.

\section{MATERIALES Y MÉTODOS}

Área de estudio: El estudio se llevó a cabo en las últimas estribaciones del Chaco Árido, hacia el oeste de Argentina, en la localidad de Chucuma, ubicada en la provincia de San Juan ( $30^{\circ} 58^{\prime} 00^{\prime}$ S \& $67^{\circ} 17^{\prime} 00^{\prime}$ ' W, $744 \mathrm{msnm}$ ), que corresponde a la distribución más occidental del Chaco Árido y pertenece a la provincia fitogeográfica del Chaco. La vegetación dominante conforma un bosque abierto xerófilo constituido por Schinopsis haenkeana, Aspidosperma quebracho-blanco, diversos algarrobos (Prosopis spp.) y Celtis ehrenbergiana. Entre los arbustos más representativos se encuentra Larrea divaricata, Mimozyganthus carinatus, Bulnesia foliosa, Prosopis torquata y numerosas gramíneas como Gounia paraguariensis, Chloris virgata, Aristida mendocina y Pappophorum caespitosum (Márquez, Ripoll, Dalmaso, Ariza, \& Jordan, 2014). La temperatura media anual es de $19.5^{\circ} \mathrm{C}$ con una mínima y máxima promedio entre 11 y $26^{\circ} \mathrm{C}$, respectivamente. El período de máxima concentración de lluvias es de noviembre a marzo, con un promedio anual de $300 \mathrm{~mm}$ (Morello, Sancholuz, Blanco, \& Protomastro, 1985).

Trabajo de campo: Los muestreos se realizaron teniendo en cuenta tres periodos definidos por la actividad reproductiva. El período reproductivo (noviembre 2014) comprende cortejo, copula y fecundación; el período de reclutamiento (febrero 2015) en el cual sólo se observaron nacimientos y el período pos-reproductivo (marzo 2015) destinado a la reserva de cuerpos grasos, sin actividad reproductiva. Cada período de muestreo tuvo la duración de un día. El horario de muestreo fue de 9:00 a 19:00 h, cubriendo el periodo de actividad diaria de la especie. Para el agrupamiento de los tres períodos se tuvo en cuenta la biología reproductiva de L. chacoensis, ya que se conoce 
que tanto en machos como hembras los eventos reproductivos son marcadamente diferentes en cada período (Galdeano, com. pers). La técnica de captura fue mediante lazo o por captura manual. Se capturó un total de 44 individuos adultos (reproductivo: 17 individuos; reclutamiento: 10 individuos y en el período posreproductivo: 17 individuos). Inmediatamente después de la captura, se registró la temperatura corporal de campo de cada lagarto insertando una sonda $0.5 \mathrm{~cm}$ en la cloaca, utilizando un termómetro de lectura rápida (TES TP-K01, $1.6 \mathrm{~mm}$, Taipei, Taiwán). Los individuos fueron manipulados por la cabeza para evitar la transferencia de calor. Registramos la LHC de cada lagarto utilizando un calibre Vernier (Lee Tools $\pm 0.02 \mathrm{~mm}$ ) y la masa corporal (MC) con una balanza digital portátil (Pesola PPS 200, \pm $0.1 \mathrm{~g})$. Se consideraron hembras adultas aquellas que presentaron una LHC mayor a $45 \mathrm{~mm}$ (Pelegrin \& Bucher, 2015) y machos adultos aquellos individuos que superaron los $37 \mathrm{~mm}$ (Cruz \& Ramírez Pinilla, 1996). El sexo se diferenció de acuerdo a los caracteres dimórficos sexuales externos (Cabrera et al., 2013). Se capturaron 21 hembras, de las cuales siete fueron identificadas como grávidas y 14 como no grávidas. El estado de gravidez de las hembras se detectó por palpación abdominal. La diferencia entre hembras grávidas y no grávidas se discriminó para el ensayo de temperatura seleccionada, ya que esta condición podría ser un factor modulador de $\mathrm{T}_{\text {sel }}$ (Labra et al., 2008). Las capturas fueron autorizadas por la Secretaría de Ambiente, Dirección de Conservación y Áreas Protegidas de la provincia de San Juan (exp. no. 004047-2012, H.J.V).

Inmediatamente después de la captura se registraron las temperaturas microambientales en cada sitio. La temperatura del sustrato $\left(\mathrm{T}_{\mathrm{s}}\right)$ se registró por contacto de la sonda del termómetro con el sustrato y la temperatura del aire $\left(T_{a}\right)$ se obtuvo a $1 \mathrm{~cm}$ de altura del sustrato. Tanto $T_{s}$ como $T_{a}$ se registraron utilizando un termómetro de lectura rápida (TES TP-K02). Por otro lado, para registrar las temperaturas operativas $\left(\mathrm{T}_{\mathrm{o}}\right)$ se utilizaron modelos biofísicos (66 x $15 \mathrm{~mm})$ cilíndricos, huecos de cloruro de polivinilo (PVC) y de coloración gris opaca, simulando el tamaño corporal ( $\sin$ cola) de un individuo adulto. Ambos extremos de los modelos fueron cubiertos con silicona líquida, dejando en uno de ellos un pequeño orificio en el centro que permite insertar en su interior una termocupla. Los modelos biofísicos de $\mathrm{T}_{\mathrm{o}}$ fueron previamente calibrados en el laboratorio, utilizando comparaciones simultáneas de temperaturas corporales de los lagartos y los modelos de PVC. El calentamiento $\left( \pm 40{ }^{\circ} \mathrm{C}\right)$ y enfriamiento $\left( \pm 10^{\circ} \mathrm{C}\right)$ se realizó por medio de una fuente artificial de calor y frío. La calibración se realizó por dos horas consecutivas (una hora para el calentamiento y una para el enfriamiento) y se utilizaron sondas tipo P-K01 para registrar cada un minuto simultáneamente las temperaturas de los modelos y las temperaturas corporales de los lagartos. El modelo de PVC de $66 \mathrm{x}$ $15 \mathrm{~mm}$ mostró un mejor ajuste (Correlación de Spearman, $\mathrm{T}_{\mathrm{c} \text { (Modelo), }} \mathrm{r}>0.9, \mathrm{P}<0.001$ ). Se utilizaron cinco modelos, cada uno fue conectado a un registrador de datos (HOBO (C) ProV2) previamente programado para que registre las temperaturas de los micrositios, cada cinco min, durante diez horas (de 9:00 a 19:00 h). El período de tiempo de registro de las $T_{0}$ fue elegido teniendo en cuenta el rango de actividad de los lagartos (Martínez, com. pers). Para cada micrositio (bajo árbol, bajo arbusto, bajo roca, sobre roca y suelo desnudo) se colocó un modelo de PVC. Para la selección de los micrositios se consideraron los más utilizados por L. chacoensis (obs. pers.).

Trabajo de laboratorio: Los individuos capturados fueron trasladados al laboratorio de biología de la FCEFyN-UNSJ, para estimar la $\mathrm{T}_{\text {sel }}$. Este ensayo se realizó siguiendo el protocolo estandarizado de Hertz et al. (1993). Se utilizó un terrario de madera con ocho subdivisiones con el fin de colocar cada individuo en un carril independiente. Se logró un gradiente lineal de temperatura $\left(17-65^{\circ} \mathrm{C}\right)$ en concordancia con los estudios de Ibargüengoytía, Renner, Boretto, Piantoni y Cussac (2007), Kubisch, Fernández e Ibargüengoytía (2011, 
2016). El extremo caliente se generó a partir de una línea de ocho lámparas de 70 watts. El extremo frío se logró enfriando el laboratorio a $17^{\circ} \mathrm{C}$, utilizando un aire acondicionado; este extremo coincidió con la temperatura ambiente del laboratorio alcanzando un rango de temperatura aproximado de $16-18^{\circ} \mathrm{C}$. La $\mathrm{T}_{\text {sel }}$ se obtuvo al adherir a cada individuo una termocupla ultrafina conectada a un dispositivo TC-08 USB (OMEGA ${ }^{\circledR}$ Precision Fine Wire Thermocouples, diámetro $0.5 \mathrm{~mm}$ ). La termocupla se colocó en el lado ventral de los ejemplares, adhiriéndola mediante una cinta adhesiva hipoalergénica. Cada individuo estuvo durante tres horas en este ensayo sin interferir ni manipular a los mismos durante el registro. Con los valores de $\mathrm{T}_{\text {sel }}$ de cada individuo se obtuvo un valor promedio. Se calculó el rango intercuartil de $\mathrm{T}_{\text {sel }}$ con los cuartiles del 25 y $75 \%\left(\mathrm{~T}_{\text {sel- }}\right.$ inferior $\mathrm{y} \mathrm{T}_{\text {sel-superior }}$ ), que representa los límites naturales en los que funciona el termostato hipotalámico (Hertz et al.,1993). Ninguno de los individuos murió durante el estudio y una vez culminados los ensayos fueron regresados al sitio de estudio.

Eficiencia en la termorregulación: Las tres variables térmicas registradas para la población $\left(\mathrm{T}_{\mathrm{c}}, \mathrm{T}_{\mathrm{sel}} \mathrm{y} \mathrm{T}_{\mathrm{o}}\right)$, fueron utilizadas para calcular la eficiencia en la termorregulación $(E)$ por medio del protocolo propuesto por Hertz et al. (1993).

Se cuantificó la media de la precisión en la termorregulación $\left(\mathrm{d}_{\mathrm{b}}\right)$, que es igual a la diferencia de la $T_{c}$ con respecto al rango intercuartil de $T_{\text {sel }}$ de cada individuo. Luego se calculó el índice de calidad térmica del ambiente desde la perspectiva de un organismo no termorregulador $\left(\mathrm{d}_{\mathrm{e}}\right)$, como la diferencia de la $\mathrm{T}_{\mathrm{o}}$ con respecto al rango intercuartil de $\mathrm{T}_{\text {sel }}$ de cada individuo. Se consideraron sólo valores absolutos. Si la $\mathrm{T}_{\mathrm{c}}$ o $\mathrm{T}_{\mathrm{o}}$ están por debajo del cuartil inferior, entonces $d_{b}=$ rango intercuartil de $T_{\text {sel }}$ inferior- $T_{c} y$ $d_{e}=$ rango intercuartil de $T_{\text {sel }}$ inferior $-T_{o}$, respectivamente. Si la $T_{c}$ o $T_{o}$ están por encima del cuartil superior, entonces $d_{b}=$ rango intercuartil de $T_{\text {sel }}$ superior- $T_{c} y_{d}=$ rango intercuartil de $\mathrm{T}_{\text {sel }}$ superior $-\mathrm{T}_{\mathrm{o}}$, respectivamente. Cuando la
$\mathrm{T}_{\mathrm{c}}$ o $\mathrm{T}_{\mathrm{o}}$ estuvo dentro del rango intercuartil de $\mathrm{T}_{\text {sel }}$ se consideró $\mathrm{d}_{\mathrm{b}} \mathrm{y} \mathrm{d}_{\mathrm{e}}$ igual a cero. Un valor alto de $\mathrm{d}_{\mathrm{b}} \mathrm{y} \mathrm{d}_{\mathrm{e}}$ indican precisión y calidad térmica baja. Siendo que un valor igual o cercano a cero brindaría una idea que los organismos son altamente precisos y el sitio donde se encuentran representa un hábitat térmicamente óptimo (Lara-Reséndiz, Díaz de la Vega-Pérez, Jiménez-Arcos, Gadsden, \& Méndez-De La Cruz, 2014). Por último, la eficiencia en la termorregulación $(E)$ fue calculada como: $E=$ 1-( media $\mathrm{d}_{\mathrm{b}} /$ media $\left.\mathrm{d}_{\mathrm{e}}\right)$, siguiendo a Hertz et al. (1993). Cuando el valor de $E=1$ indica termorregulación activa, un valor de $E=0.5$ indica un comportamiento termoconforme moderado y valores de $E=0$ indican un comportamiento de termoconforme absoluto. Los valores negativos de $E$ representarían a aquellos individuos que evitan hábitats térmicamente favorables (Hertz et al., 1993).

Para evaluar el efecto de las variables microambientales sobre la temperatura corporal de campo de los individuos, se aplicaron Modelos Lineales Generalizados (GLM) con distribución de errores gaussiana y la función de enlace identity. La variable respuesta fue la $T_{c} y$ las explicativas fueron $T_{s}, T_{a}$, LHC y $\mathrm{MC}$; teniendo en cuenta el período y el sexo (Crawley, 2007). Para conocer el efecto del período, sexo y LHC sobre $\mathrm{T}_{\text {sel}}$, se utilizó un GLM con distribución gamma y función de enlace inverse. Para evaluar la significancia del período y sexo, que fueron tomadas para ambos GLM como variables categóricas, se utilizó la prueba de Wald (Sokal \& Rohlf, 1995). No obstante, la masa corporal se correlacionó con LHC $(r>0.7, \mathrm{P}<0.001)$ y por lo tanto se eliminó a MC de los modelos (Neter, Wasserman, \& Kutner, 1990). Esta selección de modelos se realizó por reducción de la devianza, como una medida del ajuste del modelo a los datos (Cayuela, 2011).

Para evaluar la variación de la $\mathrm{T}_{\mathrm{c}}$ entre período y sexo, se realizó un análisis de la varianza (ANOVA) bifactorial con interacción. Se utilizó como test a posteriori la prueba de Tukey HSD. Se probó la normalidad mediante la prueba de Shapiro-Wilks y la homogeneidad 
de varianza se analizó a través de la prueba de Bartlett. Para realizar la comparación entre las variables $\mathrm{T}_{\mathrm{s}} \mathrm{y} \mathrm{T}_{\mathrm{a}}$, se utilizó la prueba de MannWhitney, debido a que no se cumplieron los supuestos de normalidad y homogeneidad de varianza. Para observar el efecto del período y sexo sobre $\mathrm{T}_{\mathrm{s}} \mathrm{y}_{\mathrm{a}}$, se utilizó ANOVA bifactorial con interacción y como test a posteriori la prueba de Tukey HSD. Este último procedimiento también se realizó para analizar el efecto de los factores período y sexo sobre $\mathrm{T}_{\mathrm{a}}$. Finalmente, para comparar la $\mathrm{T}_{\mathrm{o}}$ entre las diferentes variables categóricas (período y micrositio) y a su vez la comparación de los índices $d_{b}$ $\mathrm{y}_{\mathrm{e}}$ según el período y sexo, se utilizaron las pruebas de Mann-Whitney y Kruskal Wallis. Todas las pruebas se trabajaron con un nivel de confianza del $95 \%$. Las pruebas estadísticas se realizaron con el software $\mathrm{R}$, versión 3.2.2 ( $\mathrm{R}$ Core Team, 2015) y SigmaPlot 11.0.

\section{RESULTADOS}

Los resultados de $\mathrm{T}_{\mathrm{c}}, \mathrm{T}_{\text {sel }}$, así como las $\mathrm{T}_{\mathrm{a}}, \mathrm{T}_{\mathrm{s}}, \mathrm{d}_{\mathrm{b}}, \mathrm{d}_{\mathrm{e}} \mathrm{y} E$ se muestran en la Tabla 1. El modelo obtenido con respecto a la relación de la $T_{c}$ de los lagartos y las variables $T_{a}, T_{s}$, LHC, período y sexo; incluyó a la $\mathrm{T}_{\mathrm{a}}$ como la única variable significativa $(\mathrm{P}<0.001)$. Este modelo muestra que la $\mathrm{T}_{\mathrm{a}}$ explicó un $62 \%$ de la variación de $\mathrm{T}_{\mathrm{c}}$ de los lagartos. La ecuación de regresión logística en el modelo seleccionado muestra que la $\mathrm{T}_{\mathrm{c}}$ aumenta $1{ }^{\circ} \mathrm{C}$ cada $0.4{ }^{\circ} \mathrm{C}$ de la $\mathrm{T}_{\mathrm{a}}$ (Fig. 1).

La temperatura corporal de campo para el período pos-reproductivo fue $3.86{ }^{\circ} \mathrm{C}$ menor con respecto a la $\mathrm{T}_{\mathrm{c}}$ del período reproductivo y $2.84{ }^{\circ} \mathrm{C}$ inferior a la $\mathrm{T}_{\mathrm{c}}$ del período de reclutamiento (ANOVA, $\mathrm{F}_{2,42}=17.05, \mathrm{P}<0.001$, Tabla 1, Fig. 2A). El sexo no tuvo un efecto significativo sobre $\mathrm{T}_{\mathrm{c}}$ (ANOVA, $\mathrm{F}_{1,43}=1.0$,

TABLA 1

Temperatura corporal de campo, temperaturas microambientales, temperatura seleccionada en laboratorio, rango intercuartil de $\mathrm{T}_{\text {sel }} \mathrm{y}$ los índices de termorregulación para la muestra total, período y sexo

TABLE 1

Field body temperature, microenvironmental temperatures, temperature selected in the laboratory, interquartile range of $\mathrm{T}_{\text {sel }}$ and the thermoregulation indices for the total sample, period and sex

\begin{tabular}{lccccccccc}
\multicolumn{1}{c}{ Clase } & $\mathrm{N}$ & $\mathrm{T}_{\text {corporal }}$ & $\mathrm{T}_{\text {aire }}$ & $\mathrm{T}_{\text {sustrato }}$ & $\mathrm{T}_{\text {seleccionada }}$ & $\begin{array}{c}\text { Rango intercuartil de } \mathrm{T}_{\text {sel }} \\
\left(\mathrm{T}_{\text {sel-inferior }} \mathrm{y} \mathrm{T}_{\text {sel-superior }}\right)\end{array}$ & $\mathrm{d}_{\mathrm{b}}$ & $\mathrm{d}_{\mathrm{e}}$ & $E$ \\
Total & 44 & $33.4 \pm 2.6$ & $28.6 \pm 4.7$ & $34.6 \pm 6.1$ & $32.8 \pm 3.5$ & $30.9 \pm 4.7-34.9 \pm 2.8$ & $2.6 \pm 3.8$ & $3.9 \pm 4.4$ & 0.3 \\
Reproductivo & 17 & $35.2 \pm 1.4$ & $32.0 \pm 2.6$ & $38.0 \pm 6.1$ & $35.3 \pm 3.3$ & $33.7 \pm 4.4-37.1 \pm 2.8$ & $1.5 \pm 1.2$ & $7.2 \pm 2.8$ & 0.7 \\
Machos & 8 & $34.3 \pm 1.5$ & $31.0 \pm 3.3$ & $36.6 \pm 6.5$ & $33.8 \pm 3.5$ & $31.5 \pm 4.3-36.1 \pm 3.2$ & $1.5 \pm 1.5$ & $8.1 \pm 3.04$ & 0.8 \\
Hembras & 9 & $35.9 \pm 0.9$ & $33.0 \pm 1.5$ & $39.2 \pm 5.9$ & $37.8 \pm 0.4$ & $37.3 \pm 0.5-38.5 \pm 0.6$ & $1.6 \pm 0.6$ & $5.7 \pm 0.3$ & 0.7 \\
Reclutamiento & 10 & $34.1 \pm 2.2$ & $30.5 \pm 3.2$ & $38.3 \pm 6.9$ & $35.5 \pm 4.1$ & $34.1 \pm 4.5-37.2 \pm 4.1$ & $0.9 \pm 1.4$ & $0.6 \pm 1.1$ & -0.5 \\
Machos & 5 & $33.5 \pm 1.7$ & $30.8 \pm 3.0$ & $37.9 \pm 9.1$ & $34.4 \pm 5.3$ & $32.6 \pm 5.8-36.5 \pm 5.4$ & $0.7 \pm 0.9$ & $1.2 \pm 1.0$ & 0.4 \\
Hembras & 5 & $34.6 \pm 2.7$ & $30.2 \pm 3.7$ & $38.7 \pm 9.7$ & $36.6 \pm 1.4$ & $35.6 \pm 1.4-38.0 \pm 1.2$ & $1.2 \pm 1.8$ & $0.06 \pm 0.1$ & -19 \\
Pos-reproductivo & 17 & $31.3 \pm 2.3$ & $24.0 \pm 3.0$ & $29.1 \pm 6.7$ & $29.8 \pm 3.1$ & $26.9 \pm 4.9-31.8 \pm 1.6$ & $4.3 \pm 5.2$ & $4.3 \pm 6.9$ & 0 \\
Machos & 10 & $31.6 \pm 1.6$ & $24.7 \pm 2.8$ & $30.3 \pm 5.0$ & $32.7 \pm 3.3$ & $30.0 \pm 4.9-35.8 \pm 1.7$ & $1.9 \pm 2.4$ & $1.1 \pm 3.6$ & -0.7 \\
Hembras & 7 & $30.6 \pm 3.0$ & $22.9 \pm 3.1$ & $27.5 \pm 6.7$ & $24.5 \pm 3.0$ & $22.3 \pm 5.1-26.0 \pm 1.6$ & $7.7 \pm 6.4$ & $9.0 \pm 7.8$ & 0.1 \\
Machos & 23 & $33.0 \pm 2.0$ & $28.2 \pm 4.3$ & $34.1 \pm 7.1$ & $33.5 \pm 3.7$ & $31.1 \pm 4.7-36.0 \pm 3.3$ & $1.5 \pm 1.9$ & $3.5 \pm 4.6$ & 0.5 \\
Hembras & 21 & $34.0 \pm 3.1$ & $29.0 \pm 5.2$ & $35.2 \pm 9.3$ & $31.8 \pm 2.7$ & $30.6 \pm 4.1-33.2 \pm 1.9$ & $4.0 \pm 5.1$ & $5.4 \pm 6.2$ & 0.2 \\
\hline
\end{tabular}

$\mathrm{T}=$ Temperatura, $\mathrm{T}_{\text {sel }}=$ Temperatura seleccionada, $\mathrm{T}_{\text {sel-inferior }}=$ Cuartil inferior de la temperatura seleccionada, $\mathrm{T}_{\text {sel-superior }}=$ Cuartil superior de la temperatura seleccionada, Media \pm desviación estándar, $d_{b}=$ Precisión en la termorregulación, $d_{e}=$ calidad térmica del hábitat, $E=$ Eficiencia en la termorregulación, $\mathrm{N}=$ Tamaño de la muestra. 


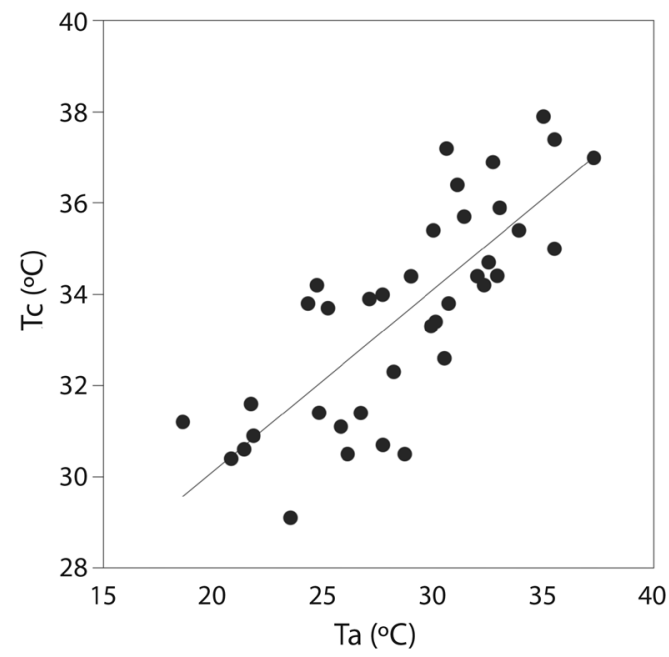

Fig. 1. Relación entre la temperatura corporal de campo $\left(\mathrm{T}_{\mathrm{c}}\right)$ de Liolaemus chacoensis y la temperatura del aire $\left(\mathrm{T}_{\mathrm{a}}\right)$, $\left(\mathrm{P}<0.001, \mathrm{~N}=44, \mathrm{~T}_{\mathrm{c}}=22.16+0.39\left(\mathrm{~T}_{\mathrm{a}}\right)\right.$ en el extremo sur del Chaco occidental, San Juan, Argentina.

Fig. 1. Relation between field body temperature $\left(T_{b}\right)$ of Liolaemus chacoensis and air temperature $\left(\mathrm{T}_{\mathrm{a}}\right),(\mathrm{P}<0.001$, $\mathrm{N}=44, \mathrm{~T}_{\mathrm{b}}=22.16+0.39\left(\mathrm{~T}_{\mathrm{a}}\right)$ in the extreme south of the western Chaco, San Juan, Argentina.

$\mathrm{P}=0.3$ ). En los tres períodos el valor promedio de $T_{c}$ se ubica dentro del rango intercuartil de $\mathrm{T}_{\text {sel }}$ (Fig. 2A). Al comparar las temperaturas microambientales $\mathrm{T}_{\mathrm{a}}$ y $\mathrm{T}_{\mathrm{s}}$ de la muestra total, se obtuvo que la $\mathrm{T}_{\mathrm{s}}$ es mayor a $\mathrm{T}_{\mathrm{a}}$ (Mann-Whitney, $\mathrm{U}=503$, Mediana $_{\mathrm{Ts}}=34.6$, Mediana $_{\mathrm{Ta}}=29.4, \mathrm{P}$ $<0.001$, Tabla 1). Las temperaturas microambientales $\mathrm{T}_{\mathrm{a}} \mathrm{y} \mathrm{T}_{\mathrm{s}}$, fueron menores en el período pos-reproductivo con respecto al período reproductivo y de reclutamiento $\left(\mathrm{T}_{\mathrm{a}}\right.$, ANOVA, $\mathrm{F}_{2,42}$ $=35.7, \mathrm{P}<0.001, \mathrm{~T}_{\mathrm{s}}$, ANOVA, $\mathrm{F}_{2,42}=8.2, \mathrm{P}=$ 0.001, Tabla 1). $\mathrm{La} \mathrm{T}_{\mathrm{a}} \mathrm{y} \mathrm{T}_{\mathrm{s}}$ donde se capturaron a las hembras en comparación con las temperaturas microambientales donde se capturaron a

Fig. 2. A. Variación de la temperatura corporal de campo $\left(\mathrm{T}_{\mathrm{c}}\right)$ de Liolaemus chacoensis en función del período (reproductivo, reclutamiento y pos-reproductivo). Las líneas continuas indican el rango intercuartil de $\mathrm{T}_{\text {sel }}$ para el período pos-reproductivo y las líneas discontinuas señalan el rango intercuartil de $\mathrm{T}_{\text {sel }}$ para el período reproductivo $\mathrm{y}$ reclutamiento. Se muestra el valor medio y la desviación estándar. B. Variación de la temperatura operativa $\left(\mathrm{T}_{\mathrm{o}}\right)$
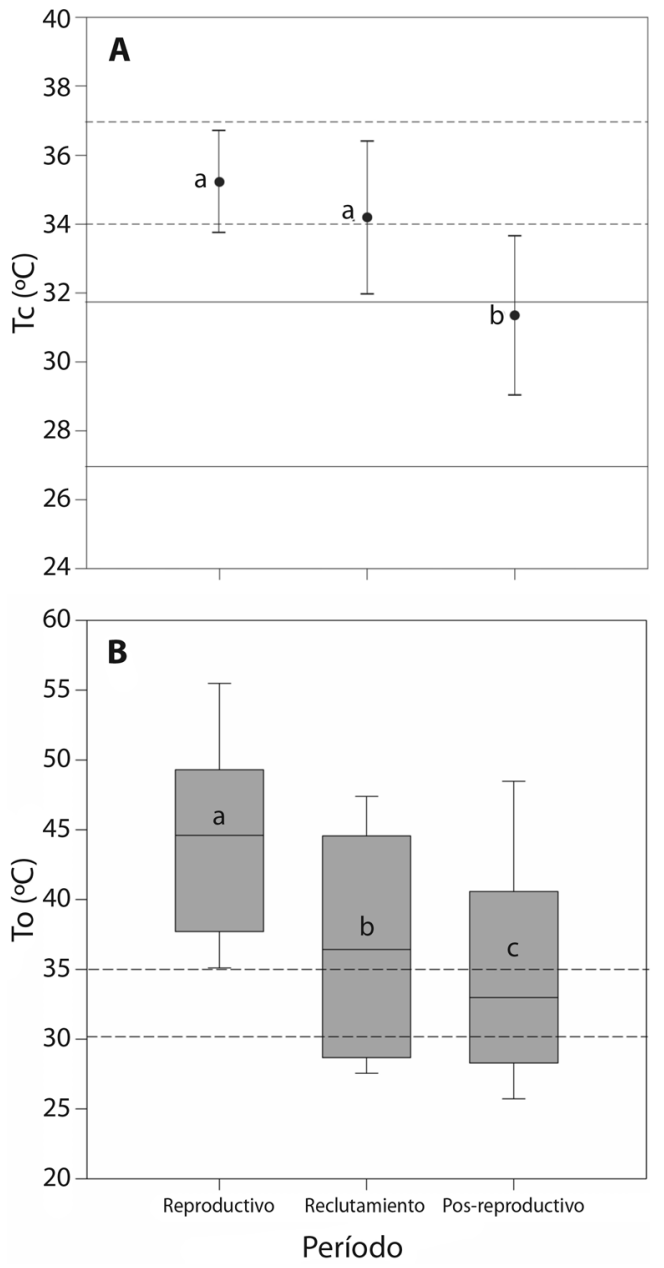

de los modelos biofísicos de acuerdo al período. La línea horizontal indica la mediana, las barras de error superior e inferior de la caja indican los percentiles 90 y 10 , respectivamente. Las líneas discontinuas indican el rango intercuartil de $\mathrm{T}_{\text {sel }}$ para la muestra total. Las letras indican diferencias significativas $(\mathrm{P}<0.05)$.

Fig. 2. A. Variation of field body temperature $\left(T_{b}\right)$ of Liolaemus chacoensis as a function of the period (reproductive, recruitment and post-reproductive). The solid lines indicate the interquartile range of $\mathrm{T}_{\text {sel }}$ for the post-reproductive period and the dashed lines indicate the interquartile range of $\mathrm{T}_{\text {sel }}$ for the reproductive and recruitment period. The mean value and the standard deviation are shown. B. Variation of the operative temperature $\left(\mathrm{T}_{\mathrm{e}}\right)$ of the biophysical models according to the period. The horizontal line indicates the median, the upper and lower error bars of the box indicate the 90th and 10th percentiles, respectively. Dashed lines indicate the interquartile range of $\mathrm{T}_{\text {sel }}$ for the total sample. The letters indicate significant differences $(\mathrm{P}<0.05)$. 
los machos fueron similares $\left(\mathrm{T}_{\mathrm{a}}\right.$, ANOVA, $\mathrm{F}_{1,43}$ $=0.02, \mathrm{P}=0.8, \mathrm{~T}_{\mathrm{s}}$, ANOVA, $\mathrm{F}_{1,43}=0.007, \mathrm{P}=$ 0.9 , Tabla 1).

La temperatura seleccionada no varió entre hembras grávidas y no grávidas (Mann-Whitney, $\mathrm{U}=33.0$; Mediana $_{\text {grávidas }}=36.5$, Mediana $_{\text {no grávidas }}=31.5, \mathrm{~N}_{\text {grávidas }}=7, \mathrm{~N}_{\text {no grávidas }}=10$, $\mathrm{P}=0.8$ ). Por lo tanto, en análisis posteriores se agruparon sólo como hembras. El modelo mínimo adecuado mostró que el sexo fue la única variable que influyó sobre la $\mathrm{T}_{\text {sel}}$, con una devianza de un $11 \%$ (Tabla 2). En hembras, la mínima temperatura seleccionada se registró a $19{ }^{\circ} \mathrm{C}$, mientras que en machos fue de $23{ }^{\circ} \mathrm{C}$. La máxima $\mathrm{T}_{\text {sel }}$ para hembras se observó a los $39{ }^{\circ} \mathrm{C}$ y para machos a $37{ }^{\circ} \mathrm{C}$. Respecto a los límites del rango intercuartil de $\mathrm{T}_{\text {sel}}$, se obtuvo que tanto el período, el sexo y la interacción de ambas variables tuvieron efecto sobre $T_{\text {sel- }}$ inferior $\mathrm{y} \mathrm{T}_{\text {sel-superior }}$ (Tabla 2). Para $\mathrm{T}_{\text {sel-inferior }}$ el modelo mínimo obtenido explica un $64 \%$ y para $\mathrm{T}_{\text {sel-superior }}$ un $62 \%$. En el período reproductivo y reclutamiento, $\mathrm{T}_{\text {sel-inferior }} \mathrm{y}_{\text {sel-superior }}$ alcanzan temperaturas por encima de los $33^{\circ} \mathrm{C}$. A diferencia del pos-reproductivo, en donde $\mathrm{T}_{\text {sel-inferior }} \mathrm{y}_{\text {sel-superior }}$ se ubican por debajo de los $32{ }^{\circ} \mathrm{C}$; particularmente, estas temperaturas fueron inferiores a $\operatorname{los} 26^{\circ} \mathrm{C}$ en hembras posreproductivas (Tabla 1 ).

$\mathrm{La} \mathrm{T}_{\mathrm{o}}$ promedio para la muestra total fue de $38.7 \pm 9.1{ }^{\circ} \mathrm{C}$ (intervalo 22.0 a $57.9^{\circ} \mathrm{C}$ ). Para el período reproductivo se obtuvo un valor de $44.3 \pm 7.2^{\circ} \mathrm{C}$ (intervalo 31.7 a $57.9^{\circ} \mathrm{C}$ ), en el de reclutamiento $37.2 \pm 7.6{ }^{\circ} \mathrm{C}$ (intervalo 26.1 a $52.2^{\circ} \mathrm{C}$ ) y en el período pos-reproductivo fue $35.0 \pm 8.6^{\circ} \mathrm{C}$ (intervalo 22.0 a $53.8^{\circ} \mathrm{C}$ ), existiendo diferencias significativas entre periodos (Kruskal-Wallis, $\mathrm{H}=937.7$, g. $1 .=2, \mathrm{P}<0.001$, Fig. 2B). La $\mathrm{T}_{\mathrm{o}}$ entre micrositios fue diferente, siendo significativamente mayor para el suelo desnudo (Kruskal-Wallis, $\mathrm{H}=2255.8$, g. 1. = $4, \mathrm{P}<0.001$, Fig. 2B). El índice de precisión en la termorregulación $\left(\mathrm{d}_{\mathrm{b}}\right)$ de $L$. chacoensis fue similar entre períodos (Kruskal-Wallis, $\mathrm{H}$ $=2.9$, g. $1 .=2, \mathrm{P}=0.2$ ) y sexo (Mann-Whitney, $\mathrm{U}=144.5, \mathrm{P}=0.1$, Tabla 1). En cuanto al índice de calidad térmica del hábitat $\left(\mathrm{d}_{\mathrm{e}}\right)$, fue significativamente diferente entre períodos (Kruskal-Wallis, $\mathrm{H}=12.3$, g. $1 .=2, \mathrm{P}=0.002$ ), observándose una mayor calidad térmica en el período de reclutamiento $\left(\mathrm{d}_{\mathrm{e}}=0.6 \pm 1.1\right)$ con respecto al reproductivo $\left(\mathrm{d}_{\mathrm{e}}=7.2 \pm 2.8\right)$ y el pos-reproductivo $\left(\mathrm{d}_{\mathrm{e}}=4.3 \pm 6.9\right)$. No obstante, no se encontraron diferencias sexuales en $d_{e}$

TABLA 2

Modelo mínimo adecuado para la temperatura seleccionada de Liolaemus chacoensis y los cuartiles inferior y superior de la temperatura seleccionada. También se indican las variables explicativas del modelo obtenido para cada variable respuesta

TABLE 2

Minimum model suitable for the selected temperature of Liolaemus chacoensis and the lower and upper quartiles of the selected temperature. The explanatory variables of the model obtained for each response variable are also indicated

\begin{tabular}{cccccc} 
Variables & Modelo & Variables explicativas & $\chi 2$ & gl & P \\
$\mathrm{T}_{\text {sel }}$ & Sexo & - & 4.7 & 1 & $0.02^{*}$ \\
$\mathrm{~T}_{\text {sel-inferior }}$ & Período + Sexo + Período*Sexo & Período & 52.2 & 3 & $<0.001^{*}$ \\
& & Sexo & 39.9 & 2 & $<0.001^{*}$ \\
& & Período*Sexo & 24.2 & 1 & $<0.001 *$ \\
$\mathrm{~T}_{\text {sel-superior }}$ & Período + Sexo + Período*Sexo & & & \\
& & Período & 38.6 & 3 & $<0.001 *$ \\
& & Sexo & 35.4 & 2 & $<0.001^{*}$ \\
& & Período*Sexo & 30.9 & 1 & $<0.001 *$ \\
\hline
\end{tabular}

$\mathrm{T}_{\text {sel }}=$ Temperatura seleccionada, $\mathrm{T}_{\text {sel-inferior }}=$ Cuartil inferior de la temperatura seleccionada, $\mathrm{T}_{\text {sel-superior }}=$ Cuartil superior de la temperatura seleccionada, $\chi 2=$ Chi-Cuadrado, $\mathrm{gl}=\operatorname{grados}$ de libertad, $\mathrm{P}=$ valor de probabilidad $(\alpha=0.05)$, asterisco $(*)$ indica diferencias significativas. 
(Mann-Whitney, $\mathrm{U}=167.0, \mathrm{P}=0.4$ ). Por otro lado, se observó que $L$. chacoensis se comporta como un termoconforme absoluto $(E=0.3)$. $\mathrm{La}$ tendencia a la termorregulación activa sólo se observó en el período reproductivo, particularmente cuando $\mathrm{d}_{\mathrm{e}}$ fue mayor a cinco y $\mathrm{d}_{\mathrm{b}}$ menor a dos (Tabla 1). La eficiencia mostro valores negativos cuando la calidad térmica del hábitat fue cercana a cero (Tabla 1).

\section{DISCUSIÓN}

Los lagartos pertenecientes al género Liolaemus tienden a tener temperaturas corporales de campo similares, independientemente de las condiciones del lugar donde habitan (Fuentes \& Jaksic, 1979; Valencia \& Jaksic, 1981; Rocha, 1995), lo cual es congruente con otros trabajos que demuestran que la biología térmica en los lagartos está filogenéticamente conservada (Bogert, 1949; Grigg \& Buckley, 2013; Clusella-Trullas \& Chown, 2014). Sin embargo, existen estudios en el género como en L. nitidus, L. lemniscatus y L. tenuis, en donde la temperatura corporal de campo depende en gran medida de las temperaturas ambientales (Labra et al., 2008). A su vez, también se observa una fisiología térmica flexible como en $L$. curis, L. leopardinus, L. montícola y L. nigroviridus (Rodríguez-Serrano, Navas, \& Bozinovic, 2009). En particular, la $T_{c}$ de $L$. chacoensis se relacionó positivamente con la $\mathrm{T}_{\mathrm{a}}$, lo que indicaría que la temperatura del aire tiene un rol importante en la termorregulación de estos individuos. La importancia de la heliotermia en la termorregulación de lagartos ha sido reportada en otras especies del género, como L. ornatus, L. islugensis y L. koslowskyi (Marquet et al., 1989; Martori et al., 2002). Por el contario, la temperatura del sustrato es elevada $\left(6^{\circ} \mathrm{C}\right.$ por encima de la $T_{a}$, Tabla 1) lo cual explicaría que la $T_{s}$ y $T_{c}$ no estén relacionadas. Asimismo, la relación $\mathrm{T}_{c}-\mathrm{T}_{\mathrm{a}}$ no se modificó entre períodos, lo que demuestra una selección activa de micrositios (Huey \& Slatkin, 1976; Huey, 1982) durante toda la actividad reproductiva. El sexo tampoco influyó en el comportamiento heliotérmico, como lo observado en los lagartos
L. tandiliensis (Stellatelli et al., 2018). Por otro lado, la temperatura corporal en $L$. chacoensis no se relacionó con LHC; como lo mencionado en otras especies de Liolaemus (Carothers, Fox, Marquet, \& Jaksic, 1997; Labra et al., 2008; Pincheira-Donoso, Hodgson, \& Tregenza, 2008; Gómez Alés, Acosta, \& Laspiur, 2017). Estas evidencias apoyan lo propuesto por Pincheira-Donoso et al. (2008), que establecen que el tamaño corporal en Liolaemus no está relacionado con la termorregulación.

La temperatura corporal de L. chacoensis registrada en campo fue de $33^{\circ} \mathrm{C}$, en coincidencia con lo mencionado por Laspiur, Villavicencio y Acosta (2007), para una población de la especie en estudio (Tabla 3). El valor de $\mathrm{T}_{\mathrm{c}}\left(33{ }^{\circ} \mathrm{C}\right)$ se encuentra dentro del rango reportado para Liolaemus, es decir, entre los 23.5 y los $37.7^{\circ} \mathrm{C}$ mencionado por Medina et al. (2012). La $\mathrm{T}_{\mathrm{c}}$ de L. chacoensis fue similar en la especie hermana L. olongasta (Cánovas, Villavicencio, Acosta, \& Marinero, 2006; Córdoba, 2018) e inferior a la $T_{c}$ de las restantes especies que conforman el clado grosseorum y que habitan en localidades del desierto de Argentina, como L. grosseorum, L. darwinii (Espinoza, Wiens, \& Tracy, 2004; Medina et al., 2012) y L. laurenti (Espinoza et al., 2004);

TABLA 3

Temperaturas corporales de campo $\left(\mathrm{T}_{\mathrm{c}}\right.$ en $\left.{ }^{\circ} \mathrm{C}\right) \mathrm{y}$ temperaturas seleccionadas $\left(\mathrm{T}_{\text {sel }}\right.$ en $\left.{ }^{\circ} \mathrm{C}\right)$ para las especies de Liolaemus del clado grosseorum

TABLE 3

Field body temperatures $\left(\mathrm{T}_{\mathrm{b}}\right.$ in $\left.{ }^{\circ} \mathrm{C}\right)$ and selected temperatures $\left(\mathrm{T}_{\text {sel }}\right.$ in $\left.{ }^{\circ} \mathrm{C}\right)$ for the Liolaemus species of the clade grosseorum

\begin{tabular}{|c|c|c|c|}
\hline Especie & $\mathrm{T}_{\mathrm{c}}$ & $\mathrm{T}_{\text {sel }}$ & Referencia \\
\hline L. chacoensis & 33.3 & & Laspiur et al., 2007 \\
\hline L. chacoensis & & 32.7 & Cruz et al., 2014 \\
\hline L. chacoensis & 33.4 & 32.8 & Este estudio \\
\hline L. darwinii & 36.4 & & Espinoza et al., 2004 \\
\hline L. darwinii & 32.8 & 33.2 & Villavicencio, 2010 \\
\hline L. darwinii & & 34.8 & Cruz et al., 2014 \\
\hline L. grosseorum & 37.3 & & Espinoza et al., 2004 \\
\hline L. laurenti & 36 & & Espinoza et al., 2004 \\
\hline L. olongasta & 32.1 & & Cánovas et al., 2006 \\
\hline L. olongasta & 34.1 & 33.8 & Córdoba, 2018 \\
\hline
\end{tabular}


estas temperaturas fueron iguales o superiores a los $36{ }^{\circ} \mathrm{C}$ (Tabla 3 ).

En los períodos, se observó el mismo patrón de variación tanto para la $\mathrm{T}_{\mathrm{c}}$ de $L$. chacoensis como para las temperaturas microambientales $\left(\mathrm{T}_{\mathrm{a}}\right.$ y $\left.\mathrm{T}_{\mathrm{s}}\right)$ siendo, mayores en el período reproductivo y de reclutamiento con respecto al pos-reproductivo (Tabla 1). Esto coincide con lo hallado por Cruz y Ramírez Pinilla (1996), donde ellos establecen que el ciclo reproductivo de L. chacoensis está modulado por la temperatura ambiental.

Por otra parte, al comparar la $\mathrm{T}_{\mathrm{c}}$ con la temperatura que los lagartos seleccionan en un gradiente térmico, L. chacoensis se encuentra activo a temperaturas corporales cercanas a sus preferencias térmicas (Fig. 2A). Estos resultados indican que esta especie obtiene los requerimientos termofisiológicos necesarios en su ambiente natural. En congruencia con lo observado para cuatro especies de Liolaemus de la Puna Argentina, L. irregularis, L. multicolor, L. albiceps y L. yanalcu (Valdecantos, Martínez, Lobo, \& Cruz, 2013).

La temperatura seleccionada fue de 32.8 ${ }^{\circ} \mathrm{C}$, la cual es similar a lo reportado por Cruz et al. (2014) para una población de L chacoensis y para el resto de las especies que conforman el clado grosseorum (Tabla 3). En particular, el sexo fue el único factor que afectó al valor promedio de $\mathrm{T}_{\text {sel }}$ en L. chacoensis, no obstante en hembras se obtuvo que la condición reproductiva no afectó la $\mathrm{T}_{\text {sel }}$. Un patrón similar se observó en L. fuscus, L. tenuis, L. montícola y L. lemniscatus (Labra \& Bozinovic, 2002). Por el contrario, en hembras vivíparas la condición de preñez si modifica a $\mathrm{T}_{\text {sel }}$ (Labra \& Bozinovic, 2002; Labra et al., 2008), como en L. curis, donde se observó que la $\mathrm{T}_{\text {sel }}$ de las hembras preñadas fue menor en comparación con las hembras no preñadas (Núñez, 1996). Con respecto al rango de temperaturas seleccionadas (inferior y superior), es importante destacar que se encontró afectado por el período y el sexo (Tabla 2). Asimismo, se observó que frente a una disminución de la actividad reproductiva y en coincidencia con una disminución de las $\mathrm{T}_{\mathrm{a}}$, en particular las hembras, alcanzaron un rango intercuartil inferior a $30{ }^{\circ} \mathrm{C}\left(22-26^{\circ} \mathrm{C}\right)$. En coincidencia con lo mencionado por Labra y Bozinovic, (2002), donde ellos establecen que un cambio en el ciclo reproductivo provoca modificaciones en los parámetros termofisiológicos. Esto demuestra que el intervalo de temperaturas seleccionadas, en ausencia de restricciones ecológicas, en hembras de $L$. chacoensis es un parámetro flexible. Por otro lado, la $T_{\text {sel }}$ no estuvo asociada al tamaño (LHC), si bien las hembras son de mayor tamaño que los machos, los cambios observados en las preferencias térmicas en L. chacoensis podrían estar relacionados a los cambios en cada evento del ciclo reproductivo y no en el tamaño.

En el período pos-reproductivo, la oferta térmica fue favorable, ya que $T_{0}$ cayó dentro del rango de temperaturas que L. chacoensis selecciona en laboratorio (Fig. 2B). No obstante, para el período reproductivo y de reclutamiento, la $T_{0}$ se ubicó fuera del rango intercuartil de $\mathrm{T}_{\text {sel. }}$ Como consecuencia, L. chacoensis podría seleccionar micrositios con alta calidad térmica como bajo árbol, aproximándose a los valores de $\mathrm{T}_{\text {sel}}$, para poder llevar a cabo sus procesos fisiológicos. Esta selección coincide con lo observado en L. koslowskyi (Martori et al., 2002), L. wiegmannii (Stellatelli, Vega, Block, \& Cruz, 2013), L. parvus (Gómez Alés et al., 2017) y en cinco especies de lagartos del sudeste de Asia (Scheffers, Edwards, Diesmos, Williams, \& Evans, 2014).

El índice de eficiencia termorregulatoria mostró que L. chacoensis se comporta como un termoconforme absoluto $(E=0.3)$, como lo observado en los lagartos de la Patagonia Argentina L. bibronii ( $E=0.4$ ) (Medina, Gutiérrez, Scolaro, \& Ibargüengoytía, 2009) y L. boulengeri $(E=0.5)$ (Medina, Scolaro, Méndez-De La Cruz, Sinervo, \& Ibargüengoytía, 2011). Por otro lado, difiere con lo reportado en el grupo goetschi estudiado por Moreno Azócar et al. (2013), donde ellos mencionan que a excepción de L. cuyanus, las especies de este grupo son termorreguladoras activas. No obstante, la estrategia termorregulatoria observada en L. chacoensis coincide con lo expresado por Huey y Slatkin (1976) y Huey 
(1982), que establecen que los animales para alcanzar las temperaturas óptimas seleccionan activamente los micrositios en su ambiente natural. Sin embargo, en el período reproductivo tanto machos y hembras cambian hacia la termorregulación activa. Este cambio podría estar influenciado por las altas $\mathrm{T}_{\mathrm{o}}$ registradas en este período (Fig. 3). Las hembras durante el período de reclutamiento evitaron micrositios térmicamente estables, alcanzando valores negativos de $E$, según Hertz et al. (1993) esto puede estar explicado por una mayor presencia de depredadores.

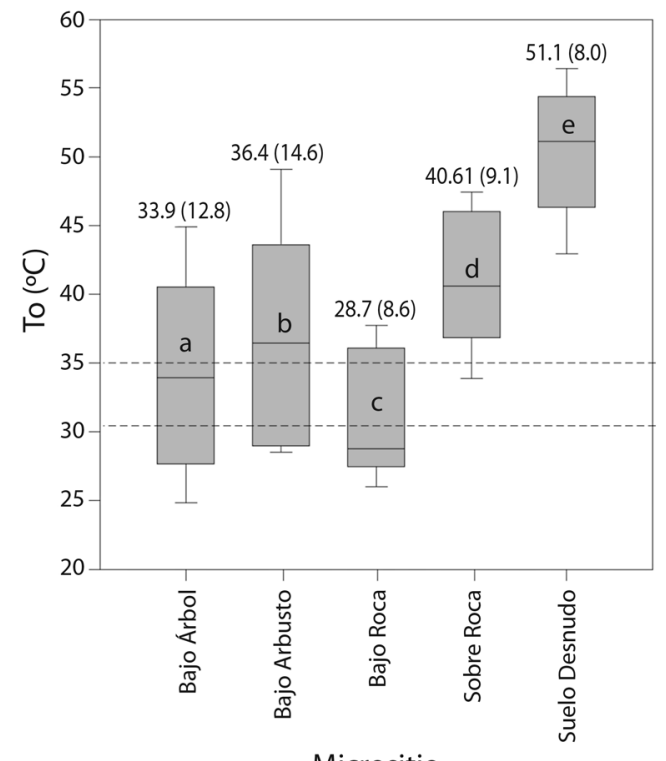

Micrositio

Fig. 3. Variación de la temperatura operativa $\left(\mathrm{T}_{\mathrm{o}}\right)$ de los modelos en los diferentes micrositios (bajo árbol, bajo arbusto, bajo roca, sobre roca y suelo desnudo), también se indican los valores de la mediana de $\mathrm{T}_{\mathrm{o}} \mathrm{y}$ entre paréntesis el rango intercuartílico. La línea horizontal indica la mediana, las barras de error superior e inferior de la caja indican los percentiles 90 y 10, respectivamente. Las líneas discontinuas indican el rango intercuartil de $\mathrm{T}_{\text {sel }}$ para la muestra total. Las letras diferentes indican diferencias significativas $(\mathrm{P}<0.05)$.

Fig. 3. Variation of the operative temperature $\left(T_{e}\right)$ of the biophysical in the different microsites (under tree, under bush, under rock, on rock and bare ground), also the values of the median $\mathrm{T}_{\mathrm{e}}$ and in parentheses the interquartile range are indicated. Horizontal line indicates the median, the upper and lower error bars of the box indicate the 90th and 10th percentiles, respectively. Dashed lines indicate the interquartile range of $\mathrm{T}_{\text {sel }}$ for the total sample. Different letters indicate significant differences $(\mathrm{P}<0.05)$.
Finalmente, es importante destacar que los parámetros termofisiológicos de L. chacoensis mostraron variación en los distintos períodos del ciclo reproductivo, cuando la temperatura ambiental comienza a elevarse. Además, mostró un comportamiento termoconforme, debido a la disponibilidad de micrositios térmicos que existen en su hábitat natural. No obstante, durante el periodo reproductivo se comportó como un termorregulador activo, ya que los micrositios térmicos presentaron una mayor $\mathrm{T}_{\mathrm{o}}$, quedando expuesto a parches más calientes; claramente la finalidad durante este período se focaliza en la búsqueda de pareja y copula.

Por consiguiente, este trabajo proporciona un marco de referencia a futuras investigaciones sobre posibles ajustes termofisiológicos a las condiciones térmicas en ambientales variables.

Declaración de ética: los autores declaran que todos están de acuerdo con esta publicación y que han hecho aportes que justifican su autoría; que no hay conflicto de interés de ningún tipo; y que han cumplido con todos los requisitos y procedimientos éticos y legales pertinentes. Todas las fuentes de financiamiento se detallan plena y claramente en la sección de agradecimientos. El respectivo documento legal firmado se encuentra en los archivos de la revista.

\section{AGRADECIMIENTOS}

Los autores agradecen al Consejo Nacional de Investigaciones Científicas y Técnicas (CONICET) por el apoyo financiero de una beca doctoral otorgada a Graciela Astudillo, Mariela Córdoba y Rodrigo Gómez Alés. A la Secretaría de Ambiente y Dirección de Conservación y Áreas Protegidas, Provincia de San Juan por los permisos otorgados para las capturas. A Jesús Pizarro y Ana Galdeano por su apoyo en campo. También a los proyectos CICITCA-UNSJ (RES. 077-2015/CS; JCA), CICITCA-UNSJ (RES. 077-2015/CS; GB) y proyecto Jóvenes Investigadores (RES. 363515/R; HJV). 


\section{RESUMEN}

La regulación de la temperatura corporal en las lagartijas juega un rol clave para la supervivencia de las especies. Su condición físiológica, en referencia a su dependencia de la temperatura ambiente, los convierte en un excelente modelo de estudio. Asimismo, los cambios en el ciclo reproductivo provocan modificaciones en los parámetros termofisiológicos. Este estudio es el primero en abordar la relación de la termorregulación con la actividad reproductiva y el sexo, para una especie de Liolaemus de la ecorregión del Chaco. El objetivo de este trabajo fue determinar cómo influye el período reproductivo, de reclutamiento y el pos-reproductivo, así como también el efecto del sexo y la longitud-hocico-cloaca sobre la termorregulación de Liolaemus chacoensis. Los individuos fueron capturados a mano en el sector Sur de Valle Fértil, provincia de San Juan, Argentina. Se capturaron un total de 44 individuos durante noviembre 2014, febrero 2015 y marzo 2015. Se registró la temperatura corporal $\left(\mathrm{T}_{\mathrm{c}}\right)$, la temperatura del sustrato $\left(\mathrm{T}_{\mathrm{s}}\right)$ y del aire $\left(\mathrm{T}_{\mathrm{a}}\right)$. También, se utilizaron modelos biofísicos para registrar la temperatura operativa. Posteriormente, en el laboratorio se evaluó la temperatura seleccionada $\left(\mathrm{T}_{\mathrm{sel}}\right)$. Luego se calculó el índice de eficiencia termorregulatoria. Los resultados indicaron que la $\mathrm{T}_{\mathrm{c}}$ de $L$. chacoensis se relacionó positivamente con la $\mathrm{T}_{\mathrm{a}}$, presentando un comportamiento heliotérmico que se mantuvo durante los períodos y el sexo. Asimismo, la $\mathrm{T}_{\mathrm{c}}$ fue mayor para el período reproductivo y de reclutamiento con respecto al pos-reproductivo. En particular, solo el sexo tuvo efecto sobre la $\mathrm{T}_{\text {sel }}$, sin embargo para el rango intercuartil de $\mathrm{T}_{\text {sel }}$ tuvo efecto tanto el período del ciclo reproductivo como el sexo. El índice de eficiencia termorregulatoria indicó que L. chacoensis es un termoconforme absoluto. Sin embargo, en el período reproductivo tanto machos como hembras cambian hacia la termorregulación activa. Estos resultados muestran que los parámetros termofisiológicos, varían en los diferentes períodos del ciclo reproductivo y la selección activa de micrositios sería un mecanismo para el mantenimiento de la temperatura corporal de la especie.

Palabras clave: temperatura, eficiencia en la termorregulación, calidad térmica, ectotermos, ciclo reproductivo.

\section{REFERENCIAS}

Abdala, C. S., \& Quinteros, A. S. (2014). Los últimos 30 años de estudios de la familia de lagartijas más diversa de Argentina. Actualización taxonómica y sistemática de Liolaemidae. Cuadernos de Herpetología, 28(2), 55-82.

Aguilar-Kirigin, A. J., \& Ocampo, M. (2011). Ampliación de la distribución de Liolaemus chacoensis (Shreve, 1948) (Iguania: Liolaemidae) en el Chaco Boliviano. Kempffiana, 7(2), 38-40.
Astudillo, G. V. (2018). Evaluación de la flexibilidad en la fisiología térmica y desempeño locomotor en Liolaemus chacoensis (Squamata: Liolaemidae) en el Chaco de San Juan (Tesis doctoral). Universidad Nacional de Cuyo, Mendoza, Argentina.

Bauwens, D., Hertz, P. E., \& Castilla, A. M. (1996). Thermoregulation in a lacertid lizard: The relative contributions of distinct behavioral mechanisms. Ecology, 77(6), 1818-1830.

Bogert, M. (1949). Thermoregulation in reptiles, a factor in evolution. Evolution, 3, 195-211.

Cabrera, M. P., Scrocchi, G. J., \& Cruz, F. B. (2013). Sexual size dimorphism and allometry in Liolaemus of the L. laurenti group (Sauria: Liolaemidae): Morphologic lability in a clade of lizards with different reproductive modes. Zoologischer Anzeiger, 252(3), 299-306.

Cánovas, M. G., Villavicencio, H. J., Acosta, J. C., \& Marinero, J. A. (2006). Dimorfismo sexual y morfometría de una población de Liolaemus olongasta (Iguania: Liolaeminae) en la Laja, Albardón, San Juan, República Argentina. Cuadernos de Herpetología, 19(2), $57-61$.

Carothers, J. H., Fox, S. F., Marquet, P. A., \& Jaksic, F. M. (1997). Thermal characteristics of ten Andean lizards of the genus Liolaemus in central Chile. Revista Chilena de Historia Natural, 70, 297-309.

Carothers, J. H., Marquet, P. A., \& Jaksic, F. M. (1998). Thermal ecology of a Liolaemus lizard assemblage along an Andean altitudinal gradient in Chile. Revista Chilena de Historia Natural, 71, 39-50.

Cayuela, L. (2011). Una introducción a R. Madrid: Universidad Rey Juan Carlos.

Cei, J. M. (1993). Reptiles del noroeste, nordeste y este de la Argentia. Herpetofauna de las selvas subtropicales, Puna y Pampas. Museo Regionale di Scienze Naturali, 14, 1-949.

Christian, K. A. (1998). Thermoregulation by the shorthorned lizard (Phrynosoma douglassi) at high elevation. Journal of Thermal Biology, 23(6), 395-399.

Christian, K. A., \& Weavers, B. W. (1996). Thermoregulation of monitor lizards in Australia: an evaluation of methods in thermal biology. Ecological Monographs, 66(2), 139-157.

Clusella-Trullas, S., \& Chown, S. L. (2014). Lizard thermal trait variation at multiple scales: A review. Journal of Comparative Physiology B: Biochemical, Systemic, and Environmental Physiology, 184, 5-21.

Córdoba, M. (2018). Performance, aclimatación y flexibilidad térmica en Liolaemus olongasta (Liolaemidae), en el Monte de San Juan (Tesis doctoral). Universidad Nacional de Cuyo, Mendoza, Argentina. 
Crawley, M. J. (2007). The R Book. New York: John Wiley and Sons Inc.

Cruz-Elizalde, R., \& Ramírez-Bautista, A. (2016). Reproductive cycles and reproductive strategies among populations of the Rose-bellied Lizard Sceloporus variabilis (Squamata: Phrynosomatidae) from central Mexico. Ecology and Evolution, 6(6), 1753-1768.

Cruz, F. B., Moreno Azócar, D. L., Bonino, M., Schulte II, J., Abdala, C. S., \& Perotti, M. G. (2014). Clima, distribución geográfica y viviparismo en especies de Liolaemus (Reptilia; Squamata): cuando las hipótesis se ponen a prueba. Ecosistemas, 23(1), 37-45.

Cruz, F. B., \& Ramírez Pinilla, M. (1996). Actividad reproductiva en el lagarto Liolaemus chacoensis (Sauria: Tropiduridae) del Chaco occidental Salta Argentina. Revista Española de Herpetología, 10, 33-39.

Díaz, R. O. (2007). Utilización de pastizales naturales. Córdoba: Brujas.

Díaz, J. A., \& Cabezas-Díaz, S. (2004). Seasonal variation in the contribution of different behavioural mechanisms to lizard thermoregulation. Functional Ecology, 18(6), 867-875.

Du, W. G., Yan, S. J., \& Ji, X. (2000). Selected body temperature, thermal tolerance and thermal dependence of food assimilation and locomotor performance in adult blue-tailed skinks, Eumeces elegans. Journal of Thermal Biology, 25(3), 197-202.

Espinoza, R. E., Wiens, J. J., \& Tracy, C. R. (2004). Recurrent evolution of herbivory in small, cold-climate lizards: Breaking the ecophysiological rules of reptilian herbivory. Proceedings of the National Academy of Sciences, 101(48), 16819-16824.

Fitch, H. S. (1970). Reproductive cycles in lizards and snakes. University of Kansas Museo of Natural History Miscellaneos Publication, 52, 1-247.

Fuentes, E. R., \& Jaksic, F. M. (1979). Activity Temperatures of Eight Liolaemus (Iguanidae) Species in Central Chile. Copeia, 3, 546-548.

Gómez Alés, R., Acosta, J. C., \& Laspiur, A. (2017). Thermal biology in two syntopic lizards, Phymaturus extrilidus and Liolaemus parvus, in the Puna region of Argentina. Journal of Thermal Biology, 68, 73-82.

Gonzales, L., Muñoz, A., \& Cortez, E. (2006). Primer reporte sobre la herpetofauna de la reserva natural "El Corbalán”, Tarija, Bolivia. Kempffiana, 2(1), 72-94.

Grbac, I., \& Bauwens, D. (2001). Constraints on temperature regulation in two sympatric Podarcis lizards during autumn. Copeia, 2001(1), 178-186.

Grigg, J. W., \& Buckley, L. B. (2013). Conservatism of lizard thermal tolerances and body temperatures across evolutionary history and geography. Biology Letters, 9(2), 20121056.

Hertz, P. E. (1992). Evaluating thermal resource partitioning. By sympatric lizards Anolis cooki and A. cristatellus: a field test using null hypotheses. Oecologia, 90(1), 127-136.

Hertz, P. E., Huey, R., \& Stevenson, R. D. (1993). Evaluating temperature regulation by field-active ectotherms: the fallacy of the inappropriate question. The American Naturalist, 142(5), 796-818.

Huey, R. B. (1982). Temperature, Physiology and the Ecology of Reptiles. En F. H. Gans, \& C. Pough (Ed.), Biology of the Reptilia (pp. 25-91). London: Academic Press.

Huey, R. B., \& Slatkin, M. (1976). Cost and benefits of lizards thermoregulation. The Quartely Reveiw of Biology, 51(3), 363-384.

Ibargüengoytía, N. R., Renner, M. L., Boretto, J. M., Piantoni, C., \& Cussac, V. E. (2007). Thermal effects on locomotion in the nocturnal gecko Homonota darwini (Gekkonidae). Amphibia Reptilia, 28(2), 235-246.

Jaksic, F. M., \& Schwenk, K. (1983). Natural History Observations on Liolaemus magellanicus, the Southernmost Lizard in the World. Herpetologica, 39(4), 457-461.

Karlin, M. S. (2012). Cambios temporales del clima en la subregión del Chaco Árido. Multequina, 21, 3-16.

Kubisch, E. L., Fernández, J. B., \& Ibargüengoytía, N. R. (2011). Is locomotor performance optimised at preferred body temperature? A study of Liolaemus pictus argentinus from northern Patagonia, Argentina. Journal of Thermal Biology, 36(6), 328-333.

Kubisch, E. L., Fernández, J. B., \& Ibargüengoytía, N. R. (2016). Vulnerability to climate warming of Liolaemus pictus (Squamata, Liolaemidae), a lizard from the cold temperate climate in Patagonia, Argentina. Journal of Comparative Physiology B: Biochemical, Systemic, and Environmental Physiology, 186(2), 243-253.

Labra, A. (1998). Selected body temperatures of seven species of Chilena Liolaemus lizards. Revista Chilena de Historia Natural, 71, 349-358.

Labra, A., \& Bozinovic, F. (2002). Interplay between pregnancy and physiological thermoregulation in Liolaemus lizards. Ecoscience, 9(4), 421-426.

Labra, A., Vidal, M., Solis, R., \& Penna, M. (2008). Ecofisiología de reptiles. En A. Labra \& M. Vidal (Eds.), Herpetología de Chile (pp. 484-516). Santiago de Chile: Science Verlang.

Lara-Reséndiz, R. A., Díaz de la Vega-Pérez, A. H., Jiménez-Arcos, V. H., Gadsden, H., \& Méndez-De 
La Cruz, F. R. (2014). Termorregulación de dos poblaciones de lagartijas simpátridas: Sceloporus lineolateralis y Sceloporus poinsettii (Squamata: Phrynosomatidae) en Durango, México. Revista Mexicana de Biodiversidad, 85, 875-884.

Laspiur, A., Villavicencio, H. J., \& Acosta, J. C. (2007). Liolaemus chacoensis (NCN). Body temperature. Herpetological Review, 38(4), 458-459.

Lavilla, E., Cruz, F. B., \& Scrocchi, G. J. (1995). Amphibiens et reptiles de la station biologique Los Colorados dans la province de Salta, Argentine. Revue Française d'Aquariologie, 22(1-2), 51-58.

Marquet, P. A., Ortíz, J. C., Bozinovic, F., \& Jaksic, F. M. (1989). Ecological aspects of thermoregulation at high altitudes: the case of andean Liolaemus lizards in northern Chile. Oecologia, 81(1), 16-20.

Márquez, J., Ripoll, Y., Dalmaso, A., Ariza, M., \& Jordan, M. (2014). Árboles Nativos de la Provincia de San Juan. San Juan, Argentina: Universidad Nacional de San Juan.

Martori, R., Aun, L., \& Orlandini, S. (2002). Relaciones térmicas temporales en una población de Liolaemus koslowskyi. Cuadernos de Herpetología, 16(1), 33-45.

Medina, M., Gutiérrez, J., Scolaro, A., \& Ibargüengoytía, N. (2009). Thermal responses to environmental constraints in two populations of the oviparous lizard Liolaemus bibronii in Patagonia, Argentina. Journal of Thermal Biology, 34, 32-40.

Medina, M., Scolaro, A., Méndez-De La Cruz, F., Sinervo, B., \& Ibargüengoytía, N. (2011). Thermal relationships between body temperature and environment conditions set upper distributional limits on oviparous species. Journal of Thermal Biology, 36, 527-534.

Medina, M., Scolaro, A., Méndez-De la Cruz, F., Sinervo, B., Miles, D. B., \& Ibargüengoytía, N. (2012). Thermal biology of genus Liolaemus: A phylogenetic approach reveals advantages of the genus to survive climate change. Journal of Thermal Biology, 37(8), 579-586.

Morello, J. H., Sancholuz, L. A., Blanco, C. A., \& Protomastro, J. (1985). Estudio macroecológico de los Llanos de la Rioja. Serie del Cincuentenario de la Administración de Parques Nacionales, 34, 242-248.

Moreno Azócar, D. L., Vanhooydonck, B., Bonino, M. F., Perotti, M. G., Abdala, C. S., Schulte, J. A., \& Cruz, F. B. (2013). Chasing the Patagonian sun: Comparative thermal biology of Liolaemus lizards. Oecologia, 171(4), 773-788.

Moyes, C. D., \& Schulte, P. M. (2007). Principios de fisiología animal. Madrid: Pearson Educación.
Neter, J., Wasserman, W., \& Kutner, M. H. (1990). Applied Linear Statistical Models. Chicago: Irwin.

Núñez, H. (1996). Autecología comparada de dos lagartijas de Chile Central. Publicación Ocasional Museo Nacional de Historia Natural (Chile), 50, 5-60.

Olivares, P., Tapia, M., Estica, V., Henriquez, Z., \& Bustos Obregon, E. (1987). Reproductive cycle of two coastal Tropidurus lizards. Microscopía Electrónica y Biología Celular, 11(2), 107-117.

Ortiz, J. C. (1981). Revision taxonomique et biologique des Liolaemus du groupe nigromaculatus (Squamata, Iguanidae) (Tesis doctoral). Universidad de Paris VII, Francia.

Pelegrin, N., \& Bucher, E. H. (2015). Activity and reproductive patterns of lizards in the Chaco of Argentina. Journal of Natural History, 49, 2693-2708.

Pianka, E. R., \& Vitt, L. J. (2003). Lizards : Windows to the Evolution of Diversity. California: University of California Press.

Pincheira-Donoso, D., Hodgson, D. J., \& Tregenza, T. (2008). The evolution of body size under environmental gradients in ectotherms: Why should Bergmann's rule apply to lizards? BMC Evolutionary Biology, 8, 68 .

Pough, F. H., Andrews, R., Cadle, J., Crump, M., Savitzhy, A., \& Wells, K. (1998). Herpetology. Upper Saddle River, N.J.: Prentice Hall.

R Core Team. (2015). R: A language and environment for statistical computing. R Foundation for Statistical Computing. Austria. Retrieved form https://www.rproject.org

Rocha, C. F. D. (1995). Ecologia termal de Liolaemus lutzae (Sauria: Tropiduridae) em uma área de Restinga do Sudeste do Brasil. Revista Brasileira de Biologia, 55(3), 481-489.

Rodríguez-Serrano, E., Navas, C. A., \& Bozinovic, F. (2009). The comparative field body temperature among Liolaemus lizards: Testing the static and the labile hypotheses. Journal of Thermal Biology, 34(6), 306-309.

Sarmiento, G. (1972). Ecological and Floristic Convergences between Seasonal Plant Formations of Tropical and Subtropical South America. The Journal of Ecology, 60(2), 367-410.

Sartorius, S. S., do Amaral, J. P., Durtsche, R. D., Deen, C. M., \& Lutterschmidt, W. I. (2002). Thermoregulatory accuracy, precision, and effectiveness in two sand-dwelling lizards under mild environmental conditions. Canadian Journal of Zoology, 80(11), 1966-1976. 
Scheffers, B. R., Edwards, D. P., Diesmos, A., Williams, S. E., \& Evans, T. A. (2014). Microhabitats reduce animal's exposure to climate extremes. Global Change Biology, 20(2), 495-503.

Sokal, R., \& Rohlf, F. (1995). Biometry: The Principles and Practice of Statistics in Biological Research. San Francisco: WH Freeman and Company.

Stellatelli, O. A., Vega, L. E., Block, C., \& Cruz, F. B. (2013). Effects on the thermoregulatory efficiency of two native lizards as a consequence of the habitat modification by the introduction of the exotic tree Acacia longifolia. Journal of Thermal Biology, 38, 135-142.

Stellatelli, O. A., Villalba, A., Block, C., Vega, L., Dajil, J. E., \& Cruz, F. B. (2018). Seasonal shifts in the thermal biology of the lizard Liolaemus tandiliensis (Squamata, Liolaemidae). Journal of Thermal Biology, 73, 61-70.

Stevenson, R. D. (1985). The Relative Importance of Behavioral and Physiological Adjustments Controlling
Body Temperature in Terrestrial Ectotherms. The American Naturalist, 126(3), 362-386.

Uetz, P. (2006). The EMBL Reptile Database. Retrieved from http://www.reptile-database.org

Valdecantos, S., Martínez, V., Lobo, F., \& Cruz, F. B. (2013). Thermal biology of Liolaemus lizards from the high Andes: Being efficient despite adversity. Journal of Thermal Biology, 38, 126-134.

Valencia, J., \& Jaksic, F. M. (1981). Relations between activity temperature and preferred temperature of Liolaemus nitidus in central Chile (Lacertilia: Iguanidae). Studies on Neotropical Fauna and Environment, 16(3), 165-167.

Villavicencio, H. J. (2010). Ecofisiología térmica de Liolaemus darwinii (Iguania: Liolaemidae) en el centro oeste del Monte Argentino (Tesis doctoral). Universidad Nacional de Río Cuarto, Córdoba, Argentina.

Zug, G., Vitt, L., \& Caldwell, J. (2001). Herpetology. San Diego: Academic Press. 